\title{
SUR LA BIOLOGIE LARVAIRE DE CYRNEA (CYRNEA) EURYCERCA Seurat, 1914 Nématode Habronème parasite du Francolin au Togo
}

\author{
C. SEUREAU* et J. C. QUENTIN** \\ (collaboration technique : K. S. Agbalo** et A. Anglo*)
}

RÉSUMÉ. La biologie larvaire du Nématode Habronème Cyrnea (Cyrnea) eurycerca Seurat, 1914, parasite du Francolin Francolinus bicalcaratus au Togo, est étudiée expérimentalement chez l'Orthoptère Acrididae Tylotropidius patagiatus Karsch.

Les trois premiers stades larvaires sont décrits et figurés. La larve infestante obtenue après deux semaines de développement à $30^{\circ} \mathrm{C}$ présente une taille importante ( $3 \mathrm{~mm}$ de longueur).

La biologie de cet Habronème est comparée à celle des autres Spirurides. Elle se singularise par :

- une pénétration asynchrone des larves de premier stade dans le tissu adipeux de l'Insecte,

- un bref séjour dans ce tissu (5 jours environ), accompagné d'une réaction d'encapsulement et suivi d'une libération active des larves de leur capsule au cours du deuxième stade larvaire,

— des larves infestantes libres et mobiles dans l'hémocoele de l'Insecte.

\section{Larval biology of Cyrnea (Cyrnea) eurycerca Seurat, 1914, a Habronemid Nematode parasite of the Double-spurred Francolin in Togo.}

SUMMARY. Larval biology of the habronemid nematode Cyrnea (Cyrnea) eurycerca Seurat, 1914, parasite of the Double-spurred Francolin Francolinus bicalcaratus, in Togo, is experimentally studied with the orthopteran Acrididae Tylotropidius patagiatus Karsch as intermediate host.

The first three larval stages are described and illustrated. Infective larvae, which occur after two weeks of development at $30^{\circ} \mathrm{C}$, are unusually large $(3 \mathrm{~mm})$.

The biology of this habronemid nematode is compared with the biology of the other Spirurids. It differs by:

- an asynchronous penetration of the first stage larvae in the insect adipose tissue,

- a short stay in this tissue (about 5 days) with a cell reaction of encapsulation, followed by an active escape of second stage larvae out of their capsule,

- free and movable infective larvae in the hemocoele of the insect.

\footnotetext{
* Laboratoire d'Histophysiologie fondamentale et appliquée, ERA-CNRS 570, Université P. et M. Curie, 12 rue Cuvier, 75005 Paris.

** Laboratoire de Zoologie, École des Sciences, Université du Bénin, BP 1515, Lomé, Togo, et Laboratoire d'Écologie animale et de Zoologie, Université d'Orléans, F 45046 Orléans Cedex.

Accepté le $\mathrm{I}_{4}$ septembre 1982.
} 
Les Francolins Francolinus bicalcaratus capturés dans la savane au nord de Lomé (Togo), sont parasités dans une proportion de $50 \%$ des individus disséqués ${ }^{1}$, par des Nématodes Habronèmes. Ces derniers sont enfoncés dans la paroi du ventricule succentorié de l'Oiseau. Il s'agit de l'espèce Cyrnea (Cyrnea) eurycerca Seurat, 1914.

Nous confirmons l'identité du parasite par la description de ses structures céphaliques et génitales et réalisons expérimentalement son cycle biologique afin de préciser la biologie larvaire encore peu connue des Habronèmes parasites d'Oiseaux.

Les spécimens sont enregistrés au M.N.H.N. de Paris sous les no Sf 287, Sf 294 et Sf 308 .

\section{Morphologie des adultes}

\section{Structures céphaliques}

Identiques chez le mâle ( fig. $1 A, B, C, D$ ) et la femelle (fig. $2 A, B, C$ ). Tête constituée par deux pseudolèvres largement dilatées, renforcées sur chaque rebord par un bourrelet cuticulaire entouré de deux dents. En profondeur, chaque pseudolèvre porte en outre trois dents disposées en éventail, bifides à leur extrémité distale et dirigées vers l'ouverture buccale. Deux lèvres, dorsale et ventrale, recouvertes par les rebords latéraux des pseudolèvres, laissent libres quatre pièces submédianes portant quatre papilles labiales et quatre papilles céphaliques. Les lèvres, dorsale et ventrale, envoient à l'intérieur de la capsule buccale une dent dorsale et une dent ventrale plus visibles sur les structures céphaliques mâles où les pseudolèvres sont moins développées. Pharynx à paroi épaisse et à lumière ovalaire (fig. $1 \mathrm{~B}$ ).

Mâle (fig. 1), structures génitales. Bourse caudale courte à extrémité arrondie à peine supérieure en longueur $(270 \mu \mathrm{m})$ à l'envergure des deux ailes $(250 \mu \mathrm{m})$. Dix paires de papilles dont trois paires préanales. Les trois dernières paires de papilles postanales sont regroupées à l'extrémité postérieure. Les papilles des sept paires antérieures sont entourées d'une zone cuticulaire dilatée. Spicule gauche 4,4 fois plus long que le spicule droit et de même épaisseur ; gubernaculum à bords externes très fortement épaissis.

Dimensions du mâle : longueur 9, I 5 à 9,35 mm, largeur $175-200 \mu \mathrm{m}$; écart des pores amphidiaux $40 \mu \mathrm{m}$, anneau nerveux, deirides et pore excréteur à 235,308 et $300 \mu \mathrm{m}$ de l'apex ; profondeur du pharynx $4^{\circ} \mu \mathrm{m}$, longueurs respectives de l'œsophage musculaire et de l'œsophage glandulaire $350-380 \mu \mathrm{m}$ et $2350-2500 \mu \mathrm{m}$; longueurs respectives des spicules droit et gauche sur trois spécimens : I 940 et $440 \mu \mathrm{m}$, I 840 et $390 \mu \mathrm{m}, \mathrm{I} 900$ et $390 \mu \mathrm{m}$; longueur du gubernaculum $65^{-}$ $75 \mu \mathrm{m}$, longueur de la queue r $25 \mu \mathrm{m}$.

Femelle (fig. 2).

Dimensions : longueur $13 \mathrm{~mm}$ à $13,8 \mathrm{~mm}$, largeur $240 \mu \mathrm{m}$; écart des pores amphidiaux $65 \mu \mathrm{m}$, anneau nerveux, pore excréteur et deirides situés respectivement à $300-315 \mu \mathrm{m}, 400-450 \mu \mathrm{m}$ et $4^{\text {IO }}-45^{\circ} \mu \mathrm{m}$ de l'apex; profondeur du pharynx $54 \mu \mathrm{m}$, longueurs respectives de l'œsophage musculaire et de l'œesophage glandulaire 420 et $2230 \mu \mathrm{m}$; vulve située de 800 à I $000 \mu \mathrm{m}$ de l'extrémité caudale, dimensions des œufs $47-53 \mu \mathrm{m} \times 25^{-27} \mu \mathrm{m}$, longueur de la queue 235 à $285 \mu \mathrm{m}$.

I. Nous remercions Monsieur Minguez, de l'agence UTA, et Monsieur Ormand, de la Mission de coopération, qui nous ont aidés à capturer les Francolins et mis obligeamment à notre disposition les tubes digestifs des Oiseaux. 

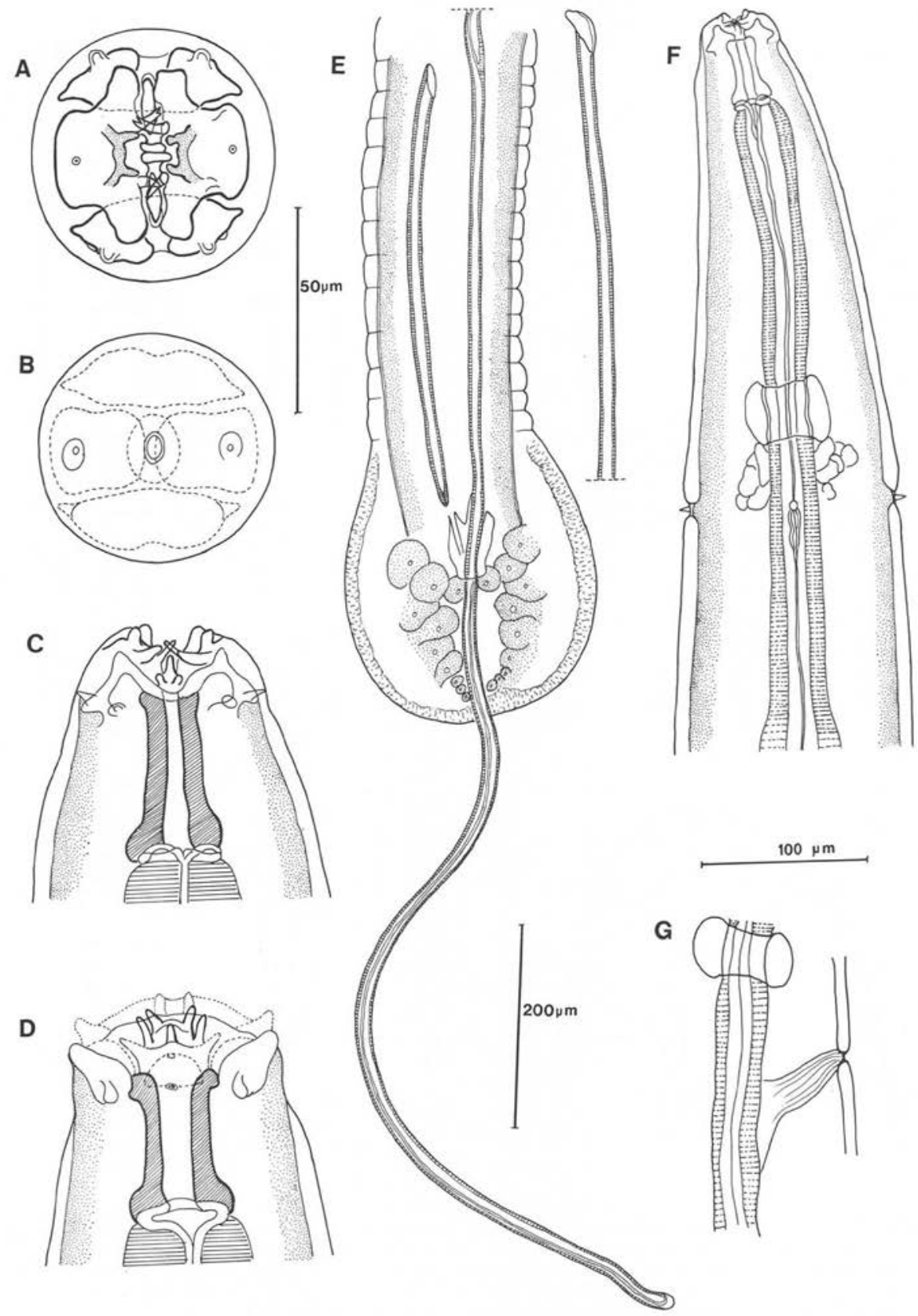

FIG. I. - Cyrnea (Cyrnea) eurycerca mâle. A, B, C et D : tête du mâle représentée successivement en vue apicale, en coupe optique au niveau du pharynx, en vue dorsale et en vue latérale ; $\mathrm{E}$ : bourse caudale représentée en vue ventrale, $\mathrm{F}$ : extrémité antérieure, région de l'œsophage musculaire, vue ventrale ; $\mathrm{G}$ : détail du pore excréteur, vue latérale.

A, B, C et D éch. $50 \mu \mathrm{m}$; E éch. $200 \mu \mathrm{m}$; F et $\mathrm{G}$ éch. 1oo $\mu \mathrm{m}$. 

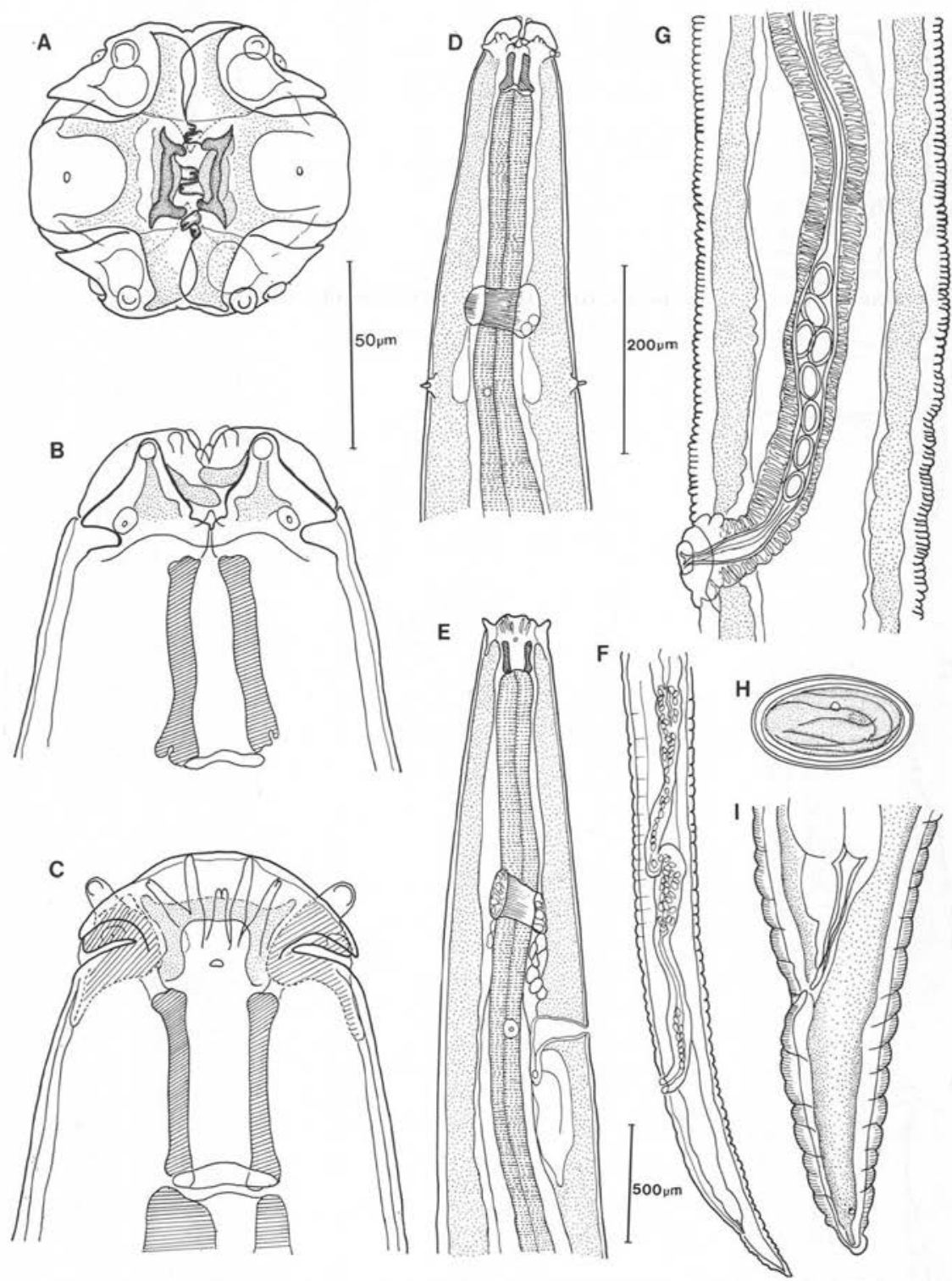

FIG. 2. - Cyrnea (Cyrnea) eurycerca femelle. A, B et C : tête de la femelle représentée successivement en vue apicale, dorsale et latérale; $\mathrm{D}$ et $\mathrm{E}$ : extrémité antérieure représentée successivement en vue ventrale et latérale ; F : région postérieure du corps avec ovéjecteur et anus; $\mathrm{G}$ : détail de l'ovéjecteur, vue latérale ; $\mathrm{H}$ : œuf ; I : détail de la région caudale.

$\mathrm{A}, \mathrm{B}, \mathrm{C}$ et H éch. $50 \mu \mathrm{m}$; D, E, G et I éch. $200 \mu \mathrm{m}$; F éch. $500 \mu \mathrm{m}$. 


\section{Identification}

Nous identifions le Nématode Habronème parasite du Francolin Francolinus bicalcaratus au Togo, à l'espèce Cyrnea (Cyrnea) eurycerca Seurat, 1914. Ses structures céphaliques correspondent en effet à la description qu'en donne Chabaud (1958) d'après des spécimens récoltés par Seurat chez la Perdrix Cacabis petrosa en Algérie. Par ailleurs, le dessin de la bourse caudale du mâle et les dimensions des mâles et des femelles concordent avec les dessins et les dimensions donnés par Seurat (1914) d'après les Cyrnea eurycerca chez la Perdrix rouge de Corse et chez la Perdrix de roche en Algérie (Seurat, 1917).

\section{Cycle biologique}

Les œufs embryonnés du parasite sont placés entre les pièces buccales d'Orthoptères Acrididae appartenant à l'espèce Tylotropidius patagiatus Karsch ${ }^{1}$, placés en élevage à une température de $30^{\circ} \mathrm{C}$.

\section{1 - Caractéristiques du développement. Chronologie des mues.}

24 heures après l'infestation, les larves sont récoltées dans l'hémocoele. Ces larves vont s'épaissir progressivement jusqu'au quatrième jour de l'infestation. Elles présenteront ensuite un développement très inégal selon qu'elles pénètreront ou non dans le tissu adipeux, ainsi que le mettront en évidence dans la suite de ce travail l'organogenèse, la croissance larvaire et l'étude histopathologique. Au début du développement, de nombreuses larves restent dans l'hémocoele. La cellule R1 subit deux divisions puis l'organogenèse s'arrête avant la formation des cordons mésodermiques et la croissance de l'intestin. Vers le cinquième jour, certaines larves pénètrent dans le tissu adipeux. L'organogenèse se poursuit alors par le développement des cordons mésodermiques et la croissance de l'intestin. La première mue survient entre le sixième et dixième jour, selon la pénétration plus ou moins rapide de la larve dans le tissu adipeux de l'insecte. La larve du deuxième stade reste encapsulée au début de sa croissance puis quitte la capsule lorsqu'elle atteint 1200 à $1300 \mu \mathrm{m}$ de longueur. A dix jours, de nombreuses larves, longues de $2 \mathrm{~mm}$, sont récoltées libres dans l'hémocoele lors de la dissection. La deuxième mue survient vers le quatorzième jour. La plupart des larves sont alors libres et actives dans l'hémocoele.

\section{2 - Organogenèse et croissance larvaire.}

La larve à l'éclosion porte, ainsi que toutes les larves de Spirurides, un crochet et 7 à 8 rangées de fines épines céphaliques. L'extrémité caudale se termine par une couronne de sept aspérités cuticulaires surmontées d'une pointe terminale.

I. Nous remercions Monsieur de Grégorio, Maître-Assistant à l'Université de Pau pour la détermination des Acrididae. 
L'organogenèse du premier stade est résumée sur la figure 3. Elle est marquée essentiellement par le développement des cordons mésodermiques issus de la cellule R1. Cet important aspect du développement n'intervient qu'après la pénétration des larves dans le tissu adipeux de l'Insecte.

L'accroissement de taille des larves chez l'Insecte, est remarquable en ce qui concerne ce Spiruride. Il s'accomplit principalement durant le second stade : la larve âgée de 24 heures mesure $166 \mu \mathrm{m}$ de long et atteint $350 \mu \mathrm{m}$ vers la fin du premier stade ; le jeune second stade mesure $455 \mu \mathrm{m}$ de long alors que la larve de troisième stade atteint $3 \mathrm{~mm}$ à $3,250 \mathrm{~mm}$.

\section{Dimensions des larves et de leurs organes.}

Premier stade larvaire (fig. 3).

- larve âgée de 24 heures (fig. $3 . A$ ). Longueur 153 à $I 66 \mu \mathrm{m}$, largeur $9 \mu \mathrm{m}$; anneau nerveux, pore excréteur, cellule excrétrice situés respectivement à 50 , 65 et $69 \mu \mathrm{m}$ de l'apex, longueurs respectives du protorhabdion, de l'œesophage et de l'intestin 35,56 et $26 \mu \mathrm{m}$; distance de la cellule RI à la pointe caudale $42 \mu \mathrm{m}$; longueur des trois cellules rectales $15 \mu \mathrm{m}$, longueur de la queue $22 \mu \mathrm{m}$; - larve âgée de 76 heures ( fig. $3 B$ ). Longueur ${ }^{7} 7 \mathrm{I} \mu \mathrm{m}$, largeur 1o $\mu \mathrm{m}$; anneau nerveux, pore excréteur et cellule excrétrice situés à 45,69 et $75 \mu \mathrm{m}$ de l'apex; longueurs du protorhabdion, de l'œsophage et de l'intestin 42,52 et $29 \mu \mathrm{m}$; distance de la cellule RI à la pointe caudale $40 \mu \mathrm{m}$, longueur des cellules rectales $13 \mu \mathrm{m}$, longueur de la queue $28 \mu \mathrm{m}$;

- larve âgée de 4 jours (fig. $3 \mathrm{C}$ ). Longueur I I I $\mu \mathrm{m}$, largeur ' ${ }_{5} \mu \mathrm{m}$; anneau nerveux, pore excréteur et cellule excrétrice situés respectivement à 48,63 et $64 \mu \mathrm{m}$ de l'apex; longueurs du protorhabdion, de l'œsophage et de l'intestin ${ }^{15}, 73$ et $49 \mu \mathrm{m}$; cellules mésodermiques issues de la division de la cellule $\mathrm{RI}$ situées à $50 \mu \mathrm{m}$ de la pointe caudale, longueur des cellules rectales $20 \mu \mathrm{m}$, longueur de la queue $28 \mu \mathrm{m}$;

- larve libre âgée de 6 jours (fig. 3 D). Longueur $175 \mu \mathrm{m}$, largeur $19 \mu \mathrm{m}$; anneau nerveux, pore excréteur et cellule excrétrice situés respectivement à $5 \mathrm{I}, 65$ et $68 \mu \mathrm{m}$ de l'apex; longueurs du protorhabdion, de l'œsophage et de l'intestin 28,60 et $42 \mu \mathrm{m}$; distance des cellules mésodermiques à la pointe caudale $56 \mu \mathrm{m}$; longueur des cellules rectales $24 \mu \mathrm{m}$, longueur de la queue $28 \mu \mathrm{m}$;

- larve encapsulée âgée de 6 jours (fig. 3 E) : formation des bandelettes mésodermiques. Longueur du corps $220 \mu \mathrm{m}$, largeur $25 \mu \mathrm{m}$; anneau nerveux, pore excréteur et cellule excrétrice situés respectivement à $5 \mathrm{I}, 68$ et $75 \mu \mathrm{m}$ de l'apex, longueurs de l'œesophage et de l'intestin 78 et $63 \mu \mathrm{m}$; longueur des cordons mésodermiques 56 à $62 \mu \mathrm{m}$; longueur des cellules rectales $28 \mu \mathrm{m}$, longueur de la queue $40 \mu \mathrm{m}$.

- larve encapsulée âgée de 6 jours, fin de premier stade (fig. $3 \mathrm{G}$ ). Longueur $350 \mu \mathrm{m}$, largeur $37 \mu \mathrm{m}$; anneau nerveux et pore excréteur situés à 62 et $87 \mu \mathrm{m}$ de l'apex, longueurs de l'œsophage et de l'intestin $\mathrm{I}_{4}$ et $\mathrm{I} 65 \mu \mathrm{m}$; ébauche génitale située à $\mathrm{I} 44 \mu \mathrm{m}$ de la pointe caudale, longueur du rectum $40 \mu \mathrm{m}$, longueur de la queue $54 \mu \mathrm{m}$;

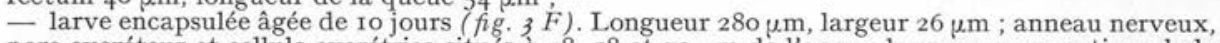
pore excréteur et cellule excrétrice situés à 48,58 et $72 \mu \mathrm{m}$ de l'apex; longueurs respectives de la région pharyngienne, de l'œsophage et de l'intestin 20,70 et $120 \mu \mathrm{m}$, ébauche génitale située à $80 \mu \mathrm{m}$ de l'apex; longueur du rectum $34 \mu \mathrm{m}$, longueur de la queue $47 \mu \mathrm{m}$.

\section{Deuxième stade lavvaire (fig. 4).}

Larves encapsulées dans des capsules à paroi mince. Dimensions des capsules $175^{-200} \mu \mathrm{m} \times$ 230-240 $\mu \mathrm{m}$ (fig. 4 A et B).

Dimensions de larves à différents stades de développement récoltées ro jours après l'infestation :

- Ire larve ( fig. ${ }_{4} C$ ). Longueur $455 \mu \mathrm{m}$, largeur $42 \mu \mathrm{m}$; anneau nerveux et pore excréteur situés à 60 et $65 \mu \mathrm{m}$ de l'apex, longueurs de l'œsophage et de l'intestin 170 et $205 \mu \mathrm{m}$; ébauche génitale située à $\mathrm{I}_{5} \mathrm{O} \mu \mathrm{m}$ de la pointe caudale, longueur des cellules rectales $50 \mu \mathrm{m}$, longueur de la queue $52 \mu \mathrm{m}$.

$\frac{52}{2} 2^{\mathrm{e}}$ larve $(f i g .4 \mathrm{D}$ ). Longueur I $280 \mu \mathrm{m}$, largeur $90 \mu \mathrm{m}$; anneau nerveux et pore excréteur situés à 65 et I75 $\mu \mathrm{m}$ de l'apex, longueur de l'œsophage $500 \mu \mathrm{m}$, longueur de l'intestin $68 \mathrm{o} \mu \mathrm{m}$, ébauche génitale située à $170 \mu \mathrm{m}$ de l'extrémité caudale, longueur de la queue $80 \mu \mathrm{m}$.

$-3^{e}$ larve, fin du deuxième stade larvaire (différenciation de l'œsophage musculaire). Longueur 2 o6o $\mu \mathrm{m}$, largeur $80 \mu \mathrm{m}$; anneau nerveux et pore excréteur situés à Ioo et $140 \% \mathrm{~m}$ de l'apex, longueur du pharynx $30 \mu \mathrm{m}$, longueurs respectives de l'œsophage musculaire, de l'œsophage glandulaire et de l'intestin I 55, 720 et I $000 \mu \mathrm{m}$; ébauche génitale située à $250 \mu \mathrm{m}$ de l'extrémité caudale, longueur du rectum $60 \mu \mathrm{m}$, longueur de la queue II $5 \mu \mathrm{m}$. 


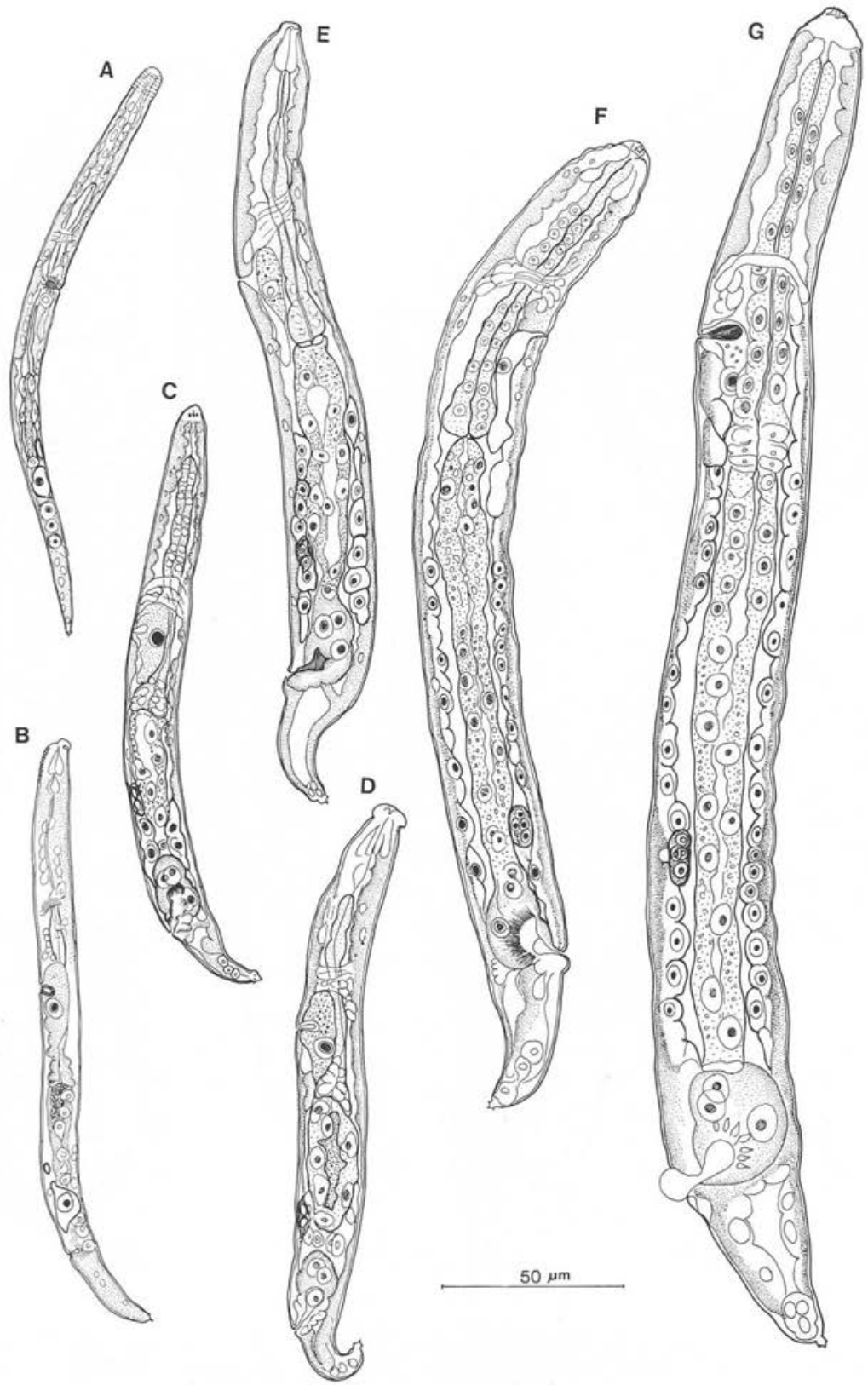

FIG. 3. - Cyrnea (Cyrnea) eurycerca premier stade larvaire. A : larve de 24 heures; B : larve de 76 heures; C, D et $\mathrm{E}$ : larves de 6 jours à différents niveaux de développement; $\mathrm{F}:$ larve de ro jours; $\mathrm{G}$ : larve âgée de 6 jours plus développée que les larves précédentes. A, B, C, D, E, F et G éch. 50 um. 


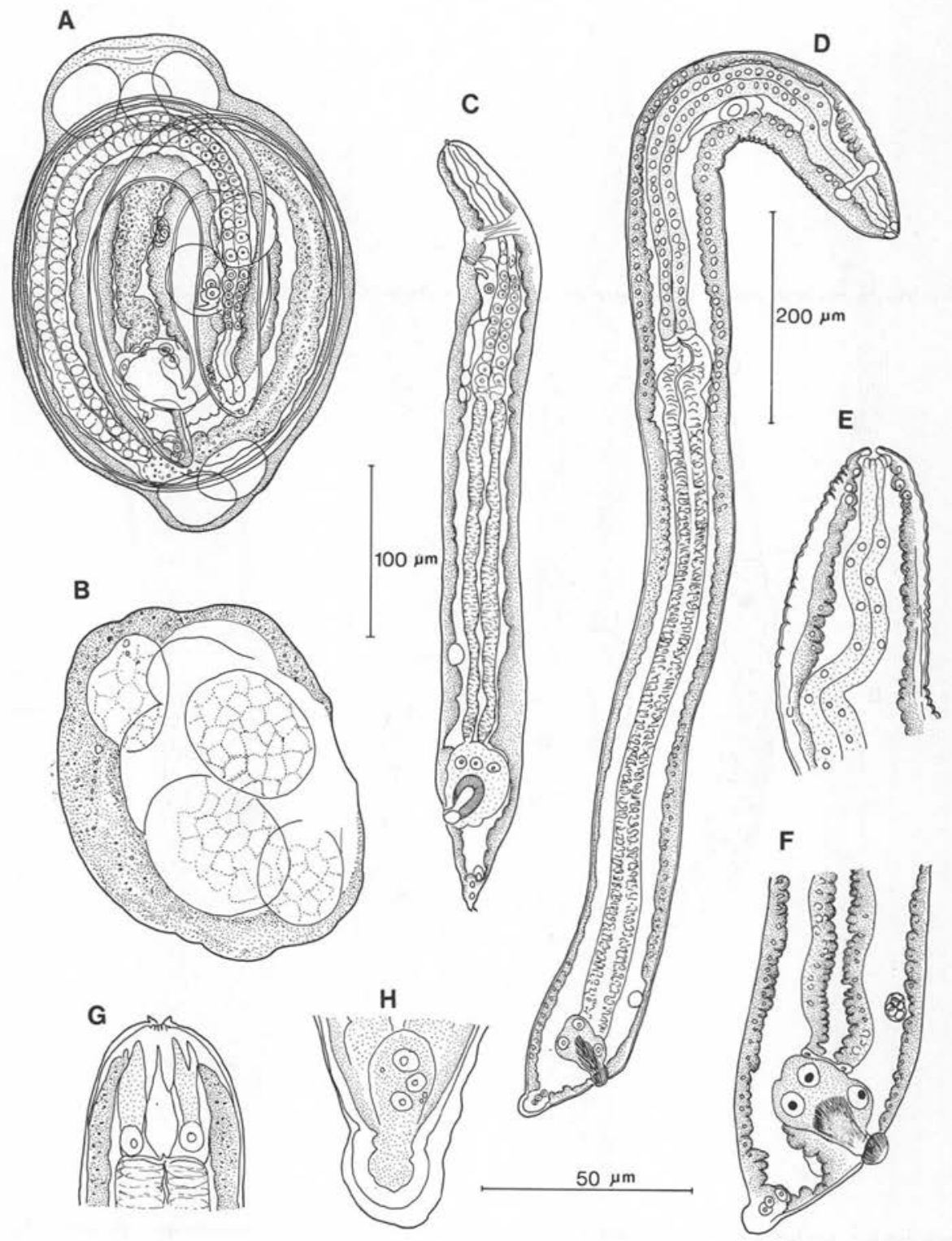

FIG. 4. - Cyrnea (Cyrnea) eurycerca deuxième stade larvaire. A : larve du second stade, Io jours après l'infestation, à l'intérieur d'une capsule formée aux dépens du tissu adipeux; noter la minceur de la paroi de la capsule ; $\mathrm{B}$ : détail d'une capsule abandonnée par la larve ; $\mathrm{C}$ : larve du second stade dégagée de la capsule mais enfermée dans l'exuvie du premier stade, ro jours après l'infestation; $\mathrm{D}$ : larve du second stade âgée de ro jours; $\mathrm{E}$ et $\mathrm{F}$ : détail des régions antérieure et rectale de cette larve; $\mathrm{G}$ et $\mathrm{H}$ : détail des régions pharyngienne et caudale. $\mathrm{A}, \mathrm{B}, \mathrm{C}, \mathrm{E}$ et $\mathrm{F}$ éch. 10o $\mu \mathrm{m}$; D éch. $200 \mu \mathrm{m} ; \mathrm{G}$ et $\mathrm{H}$ éch. $100 \mu \mathrm{m}$. 


\section{3 - Morphologie du troisième stade larvaire ( fig. 5).}

Les larves infestantes de Cyrnea eurycerca sont, au repos, courbées sur la face dorsale. Elles ne présentent pas d'ailes latérales (fig. $5 \mathrm{~A}$ ). Les structures céphaliques sont marquées par le développement d'un bourrelet cuticulaire renforçant latéralement chaque pseudolèvre et formant un cadre buccal ( fig. $5 \mathrm{~B}$ ). Chaque pseudolèvre porte sur sa face interne trois dents pointues faisant saillie en dehors de la bouche ( fig. $5 C$ et $D$ ). Les deux dents, dorsale et ventrale, caractéristiques des Habronèmes sont visibles en profondeur (fig. $5 \mathrm{E}$ ). L'intestin particulièrement développé occupe plus de la moitié de la longueur du corps. La lumière intestinale est remplie du bol alimentaire ( fig. $5 I$ ). L'extrémité caudale est arrondie et irrégulièrement garnie d'un petit nombre d'aspérités cuticulaires.

Dimensions de la larve infestante : longueur 3-3,250 mm, largeur r ro $\mu \mathrm{m}$; anneau nerveux, pore excréteur et déirides situés respectivement à $\mathrm{I} 3 \mathrm{O}-\mathrm{I} 6 \mathrm{O}, \mathrm{I}_{75-215}$ et $\mathrm{I} 3 \mathrm{O}-215 \mu \mathrm{m}$ de l'apex, longueurs respectives du pharynx, de l'œsophage musculaire, de l'œsophage glandulaire et de l'intestin $32-4 \mathrm{O}, \mathrm{I} 5 \mathrm{O}-24 \mathrm{O}, 1 \mathrm{O} 4 \mathrm{O}-\mathrm{I} 300$, I $575^{-1} 65^{\circ} \mu \mathrm{m}$; ébauche génitale située à $270-380 \mathrm{~m} \mu$ de la pointe caudale, longueur de la queue roo $\mu \mathrm{m}$.

\section{4 - Localisation et encapsulement des larves chez l'insecte.}

5 jours après l'infestation expérimentale, les larves de Cyrnea eurycerca sont, soit libres dans l'hémocoele ( fig. $6 \mathrm{~A}$ ), soit en début d'encapsulement. Dans le premier cas, les parasites sont très souvent situés à proximité de tissus tels que muscles, tissu adipeux ou épiderme. Dans le second cas, les larves ont pénétré dans un lobe de tissu adipeux qui peut alors présenter une réaction d'hypertrophie nucléaire au contact du nématode (fig. $6 \mathrm{~B}$ ). On peut aussi observer des larves à l'intérieur d'un lobe de tissu adipeux sans aucune réaction cytopathologique.

A 8 jours, quelques larves sont encore libres dans l'hémocoele, mais la plupart sont encapsulées dans le tissu adipeux. Le caractère très diffus de ce tissu chez Tylotropidius, et l'existence de fines travées d'adipocytes au contact de la plupart des organes du Criquet, expliquent un fait surprenant, la très grande diversité de répartition dans l'organisme. Toutes les larves encapsulées sont entourées d'une réaction d'hypertrophie cellulaire et surtout nucléaire particulièrement importante, certains noyaux pouvant atteindre un diamètre de 60 à $100 \mu \mathrm{m}$ (fig. $6 C$ et D). A ce stade de l'infestation, les larves ont sensiblement augmenté de taille comme le montrent les sections transversales des parasites. Toutefois, cette taille présente des différences importantes d'une larve à une autre. Ainsi, le diamètre moyen des larves, mesuré sur une vingtaine de parasites, varie de 10 à $40 \mu \mathrm{m}$. Cette hétérogénéité se retrouve aussi au niveau de la taille et de la structure des capsules. Il n'est pas rare de trouver sur une même coupe et côte à côte, deux larves de tailles très différentes, chacune dans une capsule de structure plus ou moins différenciée (fig. $6 \mathrm{D})$.

16 jours après l'infestation, des larves de grande taille (diamètre moyen : $50 \mu \mathrm{m}$ ) sont libres dans l'hémocoele, alors que d'autres, moins nombreuses, sont à l'intérieur de capsules du type de celles des Spiruridae ( fig. $7 \mathrm{~A}$ ). On observe également quelques capsules vides ( fig. $7 \mathrm{~B}$ ), et l'on est frappé par la dégradation du tissu adipeux qui 


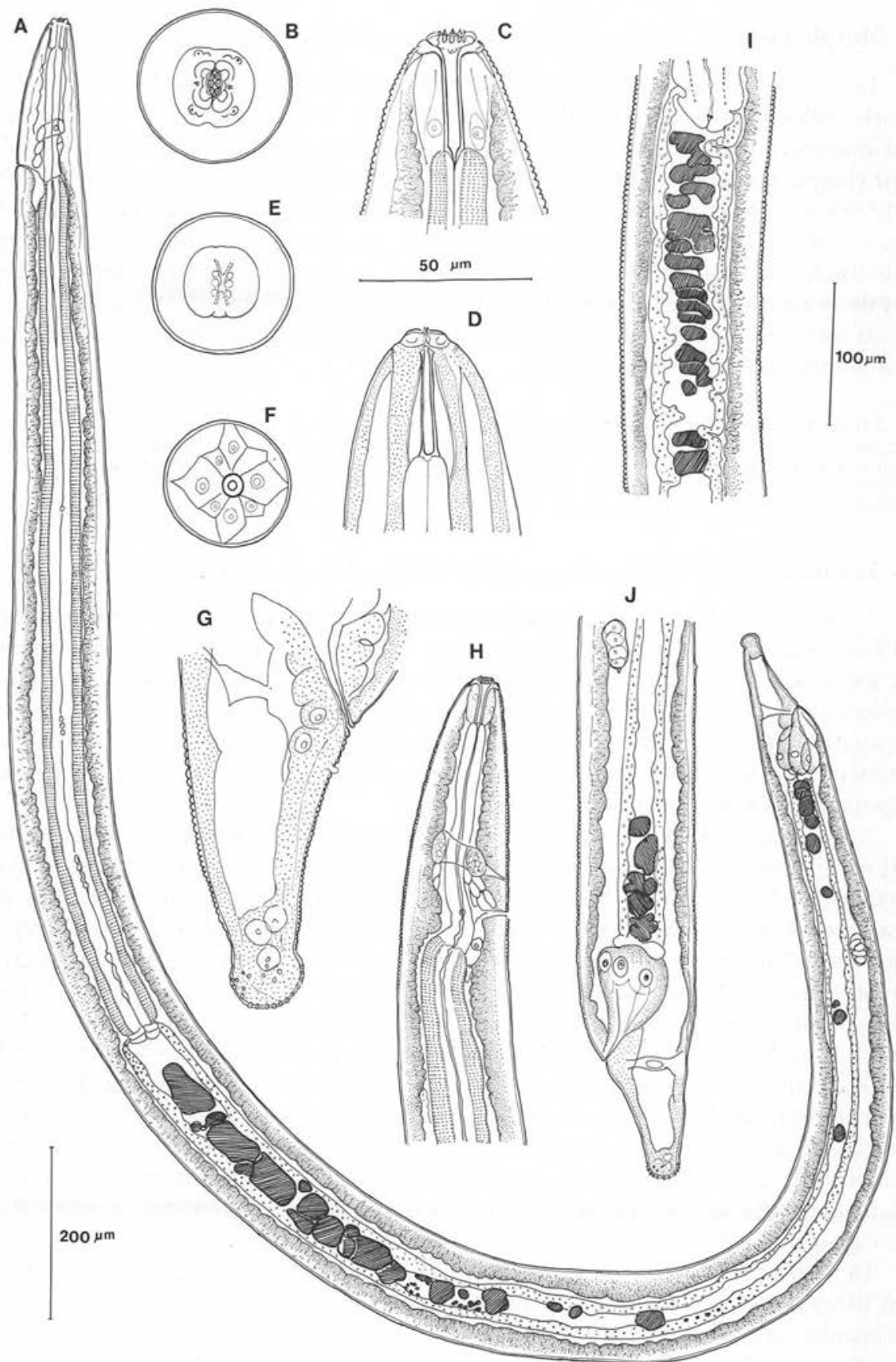

FIG. 5. - Cyrnea (Cyrnea) eurycerca troisième stade latvaire. A : larve infestante au repos, vue latérale ; B, C, D, E et F : tête représentée successivement en vue apicale, en vue latérale, en vue dorsale, en vue optique au niveau des structures labiales, en coupe optique au niveau du pharynx; $\mathrm{G}$ : détail de la région caudale ; $\mathrm{H}$ : extrémité antérieure, vue latérale ; I et $\mathrm{J}$ : régions antérieure et postérieure de l'intestin dont la lumière est remplie du bol alimentaire. A éch. $200 \mu \mathrm{m} ;$ B, C, D, E, F et G éch. $50 \mu \mathrm{m}$; H, I et J éch. $100 \mu \mathrm{m}$. 

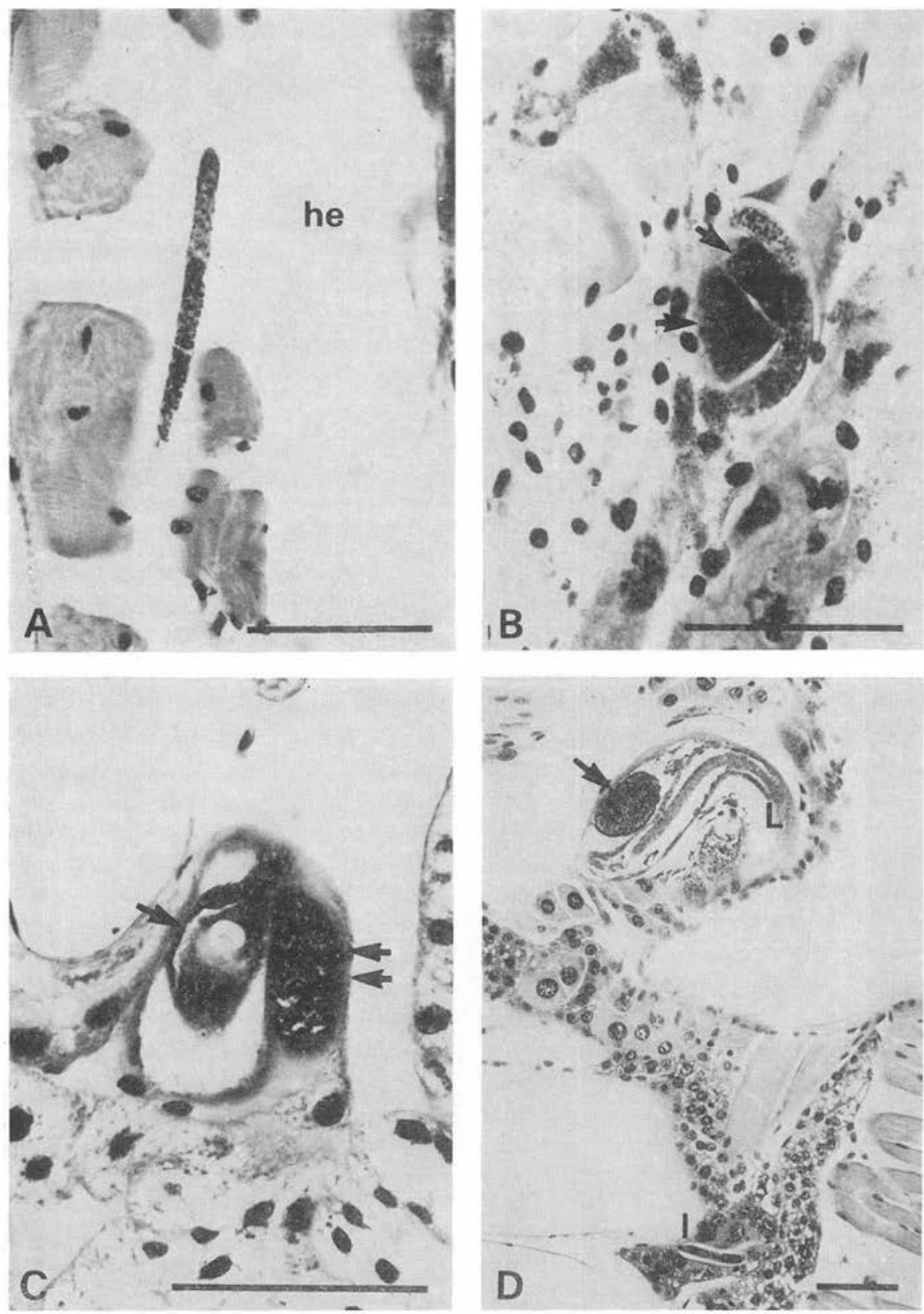

Fig. 6. - Cyrnea (Cyrnea) eurycerca, encapsulement des larves chez l'Insecte. A : 5 jours après l'infestation expérimentale, une larve est libre dans l'hémocoele (he) près de fibres musculaires ; B : 5 jours, une larve a pénétré à l'intérieur du tissu adipeux et provoqué l'hypertrophie de deux noyaux adipocytaires (flèches) ; C : 8 jours, larve encapsulée (flèche) et noyau hypertrophié (double flèche); D : 8 jours, deux larves encapsulées ( $\mathrm{L}$ et 1 ) à deux stades de développement différents, la larve L a provoqué l'hypertrophie d'un noyau (flèche) du tissu adipeux.

Échelle : roo $\mu \mathrm{m}$. 

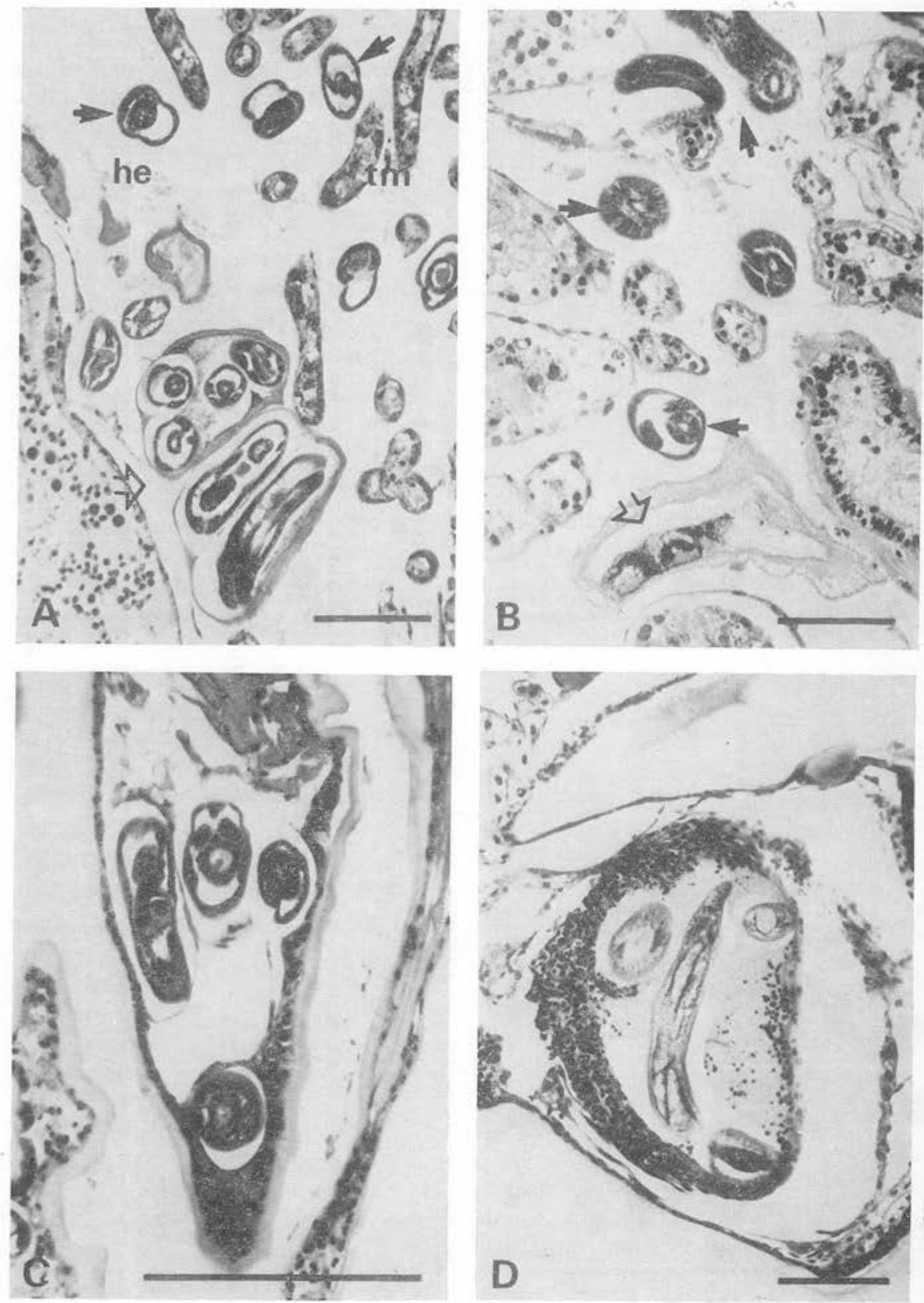

FIG. 7. - Cyrnea (Cyrnea) eurycerca, encapsulement des larves chez l'Insecte. A : I6 jours après l'infestation, des larves sont encapsulées (flèche creuse), d'autres (flèches pleines) sont libres dans l'hémocoele (he) près de tubes de Malpighi (tm); B : I6 jours, larves libres (flèches pleines) et capsule vide avec un noyau hypertrophié dégradé (flèche creuse) ; C : I6 jours, larves dans un repli tégumentaire abdominal ; D : r8 jours, larve encapsulée secondairement par des hémocytes.

Échelle : $200 \mu \mathrm{m}$. 
semble avoir été utilisé pour la nutrition des parasites. Cette observation est à mettre en relation avec l'existence d'importantes inclusions dans la lumière du tube digestif des larves infestantes. Les nématodes libres sont répartis dans tout l'organisme de l'insecte, un grand nombre d'entre eux sont logés à l'intérieur des replis tégumentaires de l'abdomen ( fig. 7 C).

On n'observe pas de réactions histopathologiques autres que celles qui ont donné naissance aux capsules d'origine adipocytaire. Cependant, un Criquet fixé 18 jours après l'infestation présente des réactions d'encapsulement hémocytaire secondaire autour de quelques larves ( fig. $7 \mathrm{D}$ ). Dans ce cas, les parasites ne présentent aucun signe de dégradation ou de lyse. Aucun dépôt pigmentaire n'est observé.

L'étude histopathologique des Criquets parasités montre donc, au cours des premiers jours qui suivent l'infestation expérimentale, un encapsulement des larves de premier stade dans le tissu adipeux, étalé dans le temps. Cet encapsulement asynchrone est caractérisé par une importante hypertrophie nucléaire des adipocytes parasités et la formation d'une capsule du type de celle des Spiruridae. Enfin, la plupart des larves quittent activement leur capsule après y avoir séjourné environ cinq jours. Elles sont alors libres dans l'hémocoele où, en général, elles ne provoquent pas de réaction cytopathologique de la part de l'hôte.

\section{Discussion}

Chez la plupart des Spirurides, la biologie de la larve préinfestante chez l'hôte intermédiaire obéit aux règles suivantes :

- les parasites pénètrent dans un tissu de l'insecte au cours du premier stade larvaire et de manière synchrone,

- les larves grandissent en même temps au cours du second stade et effectuent leur seconde mue dans le tissu où a lieu l'encapsulement,

- les larves infestantes restent emprisonnées à l'intérieur de la capsule.

La biologie du Nématode Habronème Cyrnea (Cyrnea) eurycerca diffère de ce schéma général par plusieurs aspects :

1 - Les larves pénètrent bien au cours du premier stade dans le tissu adipeux de l'insecte-hôte intermédiaire, mais de manière asynchrone. Ainsi, entre 6 et 10 jours après l'infestation, certaines larves sont libres dans l'hémocoele alors qu'au même moment, d'autres qui ont pénétré dans le tissu adipeux y ont déjà provoqué une importante réaction d'hypertrophie, premier stade de l'encapsulement.

2 - Il résulte de cette pénétration asynchrone un développement également asynchrone des larves au début du second stade qui donne naissance à des larves de tailles très différentes à un même stade de l'infestation. Cette hétérogénéité dans la taille des larves est un phénomène nouveau jamais rencontré dans les conditions expérimentales que nous utilisons, c'est-à-dire après une unique infestation expérimentale. 3 - Contrairement aux autres Spirurides que nous avons étudiés, les larves quittent leur capsule alors qu'elles sont au second stade larvaire, après y avoir séjourné en moyenne 5 à 6 jours. Ainsi, et malgré ce séjour très bref, si on le compare à la durée du 
développement larvaire, il semble que l'encapsulement des larves, de la fin du premier stade au début du second, soit déterminant pour la poursuite de l'organogenèse et notamment pour la formation des cordons mésodermiques.

Le phénomène de sortie active des larves de leur capsule explique la difficulté rencontrée par Cram (1931) pour interpréter l'absence apparente d'encapsulement des larves de Cyrnea colini chez la Blatte (Cram, 1927) : "They do not appear to encyst but develop to third stage larvae among the tissue from which they quickly emerge when the cockroach is dissected... "

Ce comportement larvaire de Cyrnea eurycerca (fin de croissance dans l'hémocoele de l'Insecte au cours du deuxième stade, mobilité du troisième stade larvaire) inhabituel chez un Spiruride a également été remarqué par Chabaud (1954) à propos du cycle d'un autre Habronème d'Oiseau, Sicarius dipterum. Chabaud interprète cette mobilité comme un des éléments ancestraux qui ont conduit aux cycles très spécialisés des Habronèmes d'Équidés (Roubaud et Decazeaux, 1921).

Remerciements : Nous remercions vivement le Professeur Johnson, Recteur de l'Université du Bénin, pour les facilités qu'il a bien voulu nous accorder dans la réalisation de ce travail.

\section{BIBLIOGRAPHIE}

Chabaud A. G. : Sur le cycle évolutif des Spirurides et de Nématodes ayant une biologie comparable. Ann. Parasitol. Hum. Comp., r954, 29, 42-88, 206-249, 358-425.

Chabaud A. G. : Essai de classification des Nématodes Habronematidae. Ann. Parasitol. Hum. Comp., 1958, 33, 445-508.

Cram E. R. : Bird parasites of the Nematode suborders Strongylata, Ascaridata and Spirurata. U.S. Natl. Mus. Bull., 1927, 140, 465 p.

CrAm E. R. : Developmental stages of some nematodes of the Spiruroidea parasitic in poultry and game birds. U.S. Dept. Agriculture Techn. Bull., 1931, 227, 1-27.

Roubaud E., Decazeaux J. : Contribution à l'histoire de la mouche domestique comme agent vecteur des Habronèmoses d'Équidés. Cycle évolutif et parasitisme de l'Habronema megastoma (Rudolphi, I8I9) chez la mouche. Bull. Soc. Pathol. Exot., 1921, I4, 471-506.

Seurat L. G. : Sur un nouveau parasite de la Perdrix rouge. C. R. Soc. Biol., 1914, 76, $390-393$.

Seurat L. G. : Nématodes de la Perdrix de roche. Bull. Soc. Hist. Nat. Afrique du Nord, 1917, 8 , 208-2I5.

Seureau C. : Réactions cellulaires provoquées par les Nématodes Subulures et Spirurides chez Locusta migratoria (Orthoptère) : localisation et structure des capsules. Z. Parasitenk., I973, $41, \mathrm{I} 19-138$.

Seureau C., Quentrn J. C. : Évolution de l'adaptation des Nématodes hétéroxènes à leur hôte intermédiaire : passage progressif d'un parasitisme extracellulaire à un parasitisme intracellulaire. C. R. Acad. Sci. Paris, 1981, 292, 421-425. 